SUPPORTING INFORMATION FOR

\title{
Resonant Tender X-ray Diffraction for Disclosing the Molecular Packing of Paracrystalline Conjugated Polymer Films
}

Guillaume Freychet, ${ }^{\dagger}$ Eliot Gann, ${ }^{\ddagger}$ Lars Thomsen, ${ }^{\S}$ Xuechen Jiao, ${ }^{\#, \nabla}$ and Christopher R. McNeill\#,*

${ }^{\dagger}$ NSLS-II, Brookhaven National Laboratory, Upton, NY 11973, USA

* Materials Measurement Science Division, Materials Measurement Laboratory, National Institute of Standards and Technology, Gaithersburg, MD 20899, USA

$\S$ Australian Synchrotron, ANSTO, 800 Blackburn Road, Clayton, VIC 3168, Australia

\# Department of Materials Science and Engineering, Monash University, Wellington Road, Clayton, VIC 3800 , Australia.
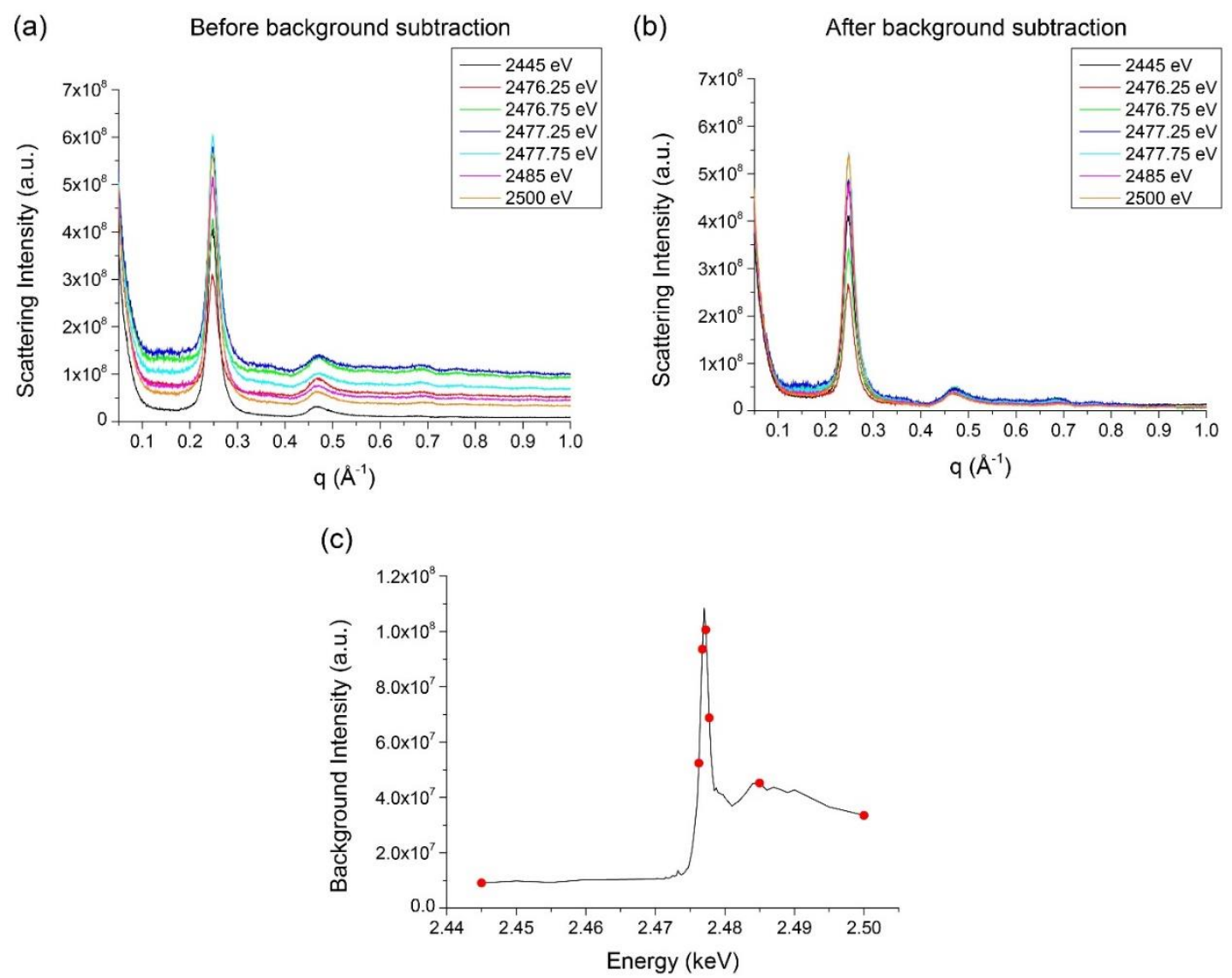

Figure S1. Demonstration of the extraction of a fluorescence yield NEXAFS spectrum from the resonant scattering data. (a) Example scattering profiles taken at select energies before background subtraction.

(b) Example scattering profiles in part (a) after background subtraction. (c) Fluorescence yield NEXAFS spectrum (line) acquired from the background intensity with the red dots showing the background intensity of the selected profiles in part (a). 


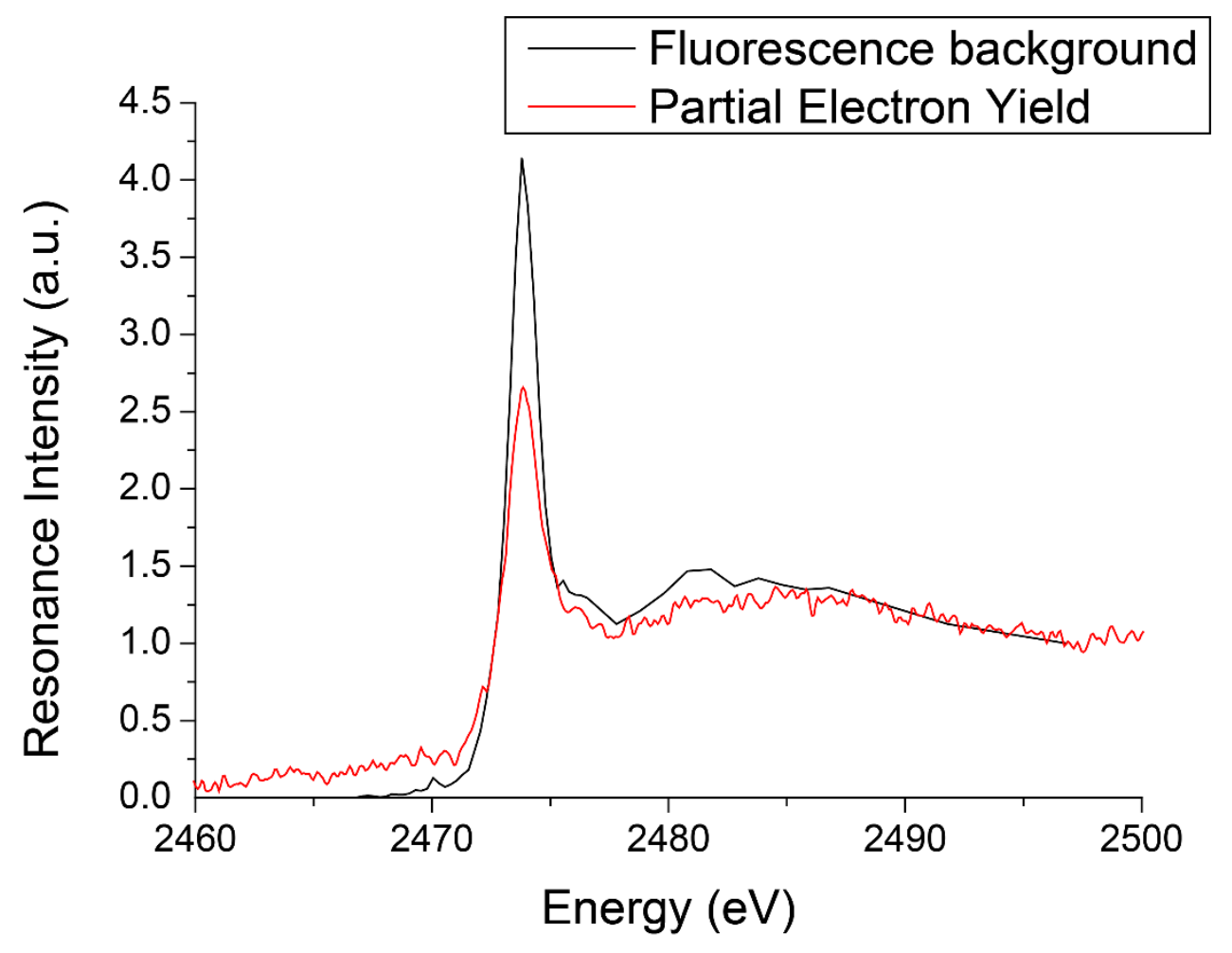

Figure S2. Comparison of fluorescence yield NEXAFS and PEY NEXAFS spectra of P(NDI2OD-T2). 
(a)

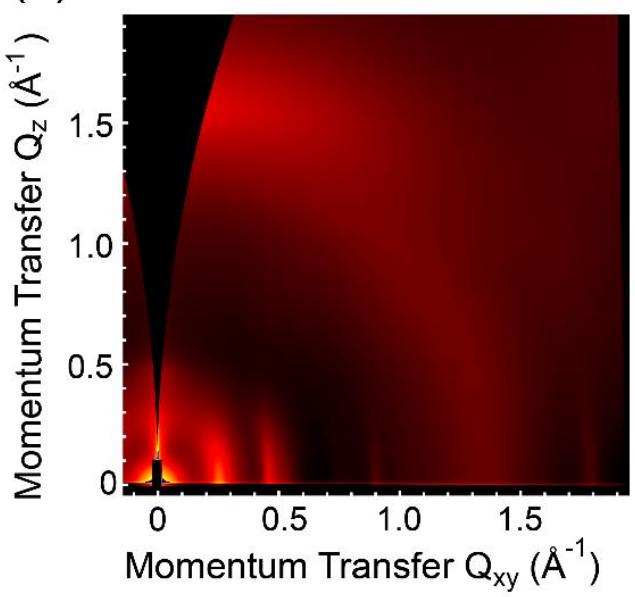

(c)

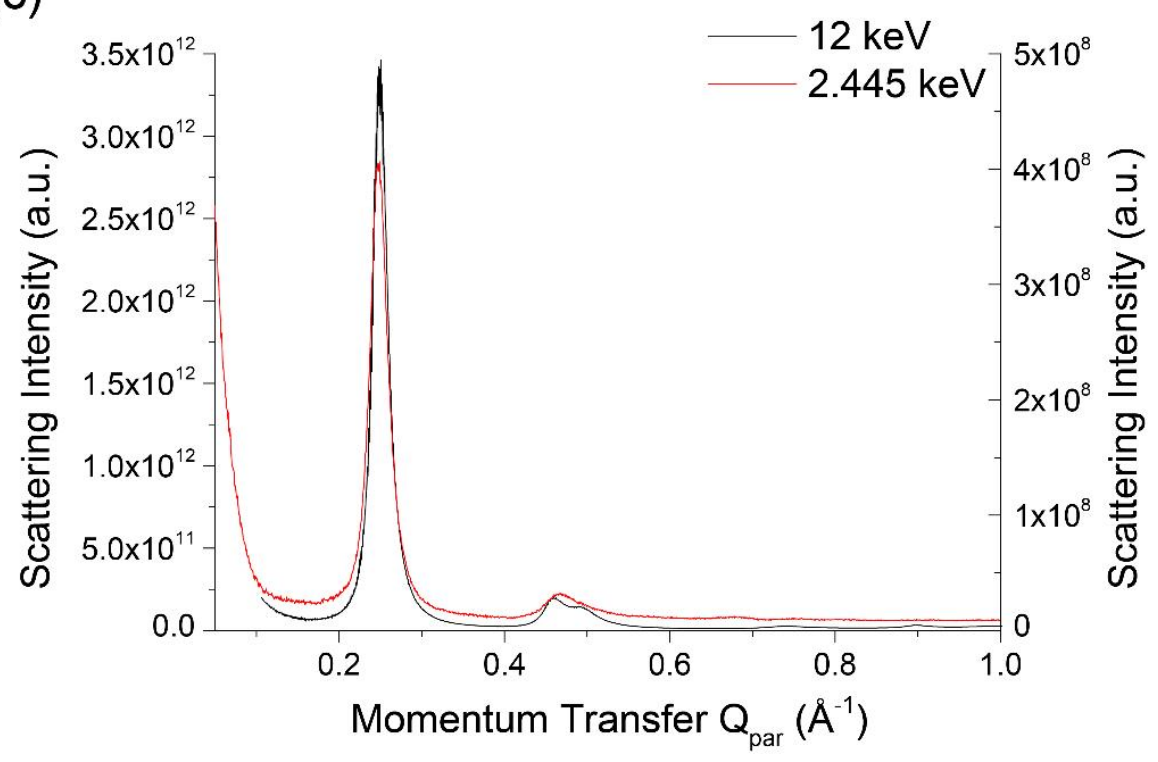

Figure S3. Comparison of GIWAXS data of P(NDI2OD-T2) taken at (a) $12 \mathrm{keV}$ and (b) $2.45 \mathrm{keV}$. (c) Comparison of $1 \mathrm{D}$ line profiles acquired at these two different energies. 


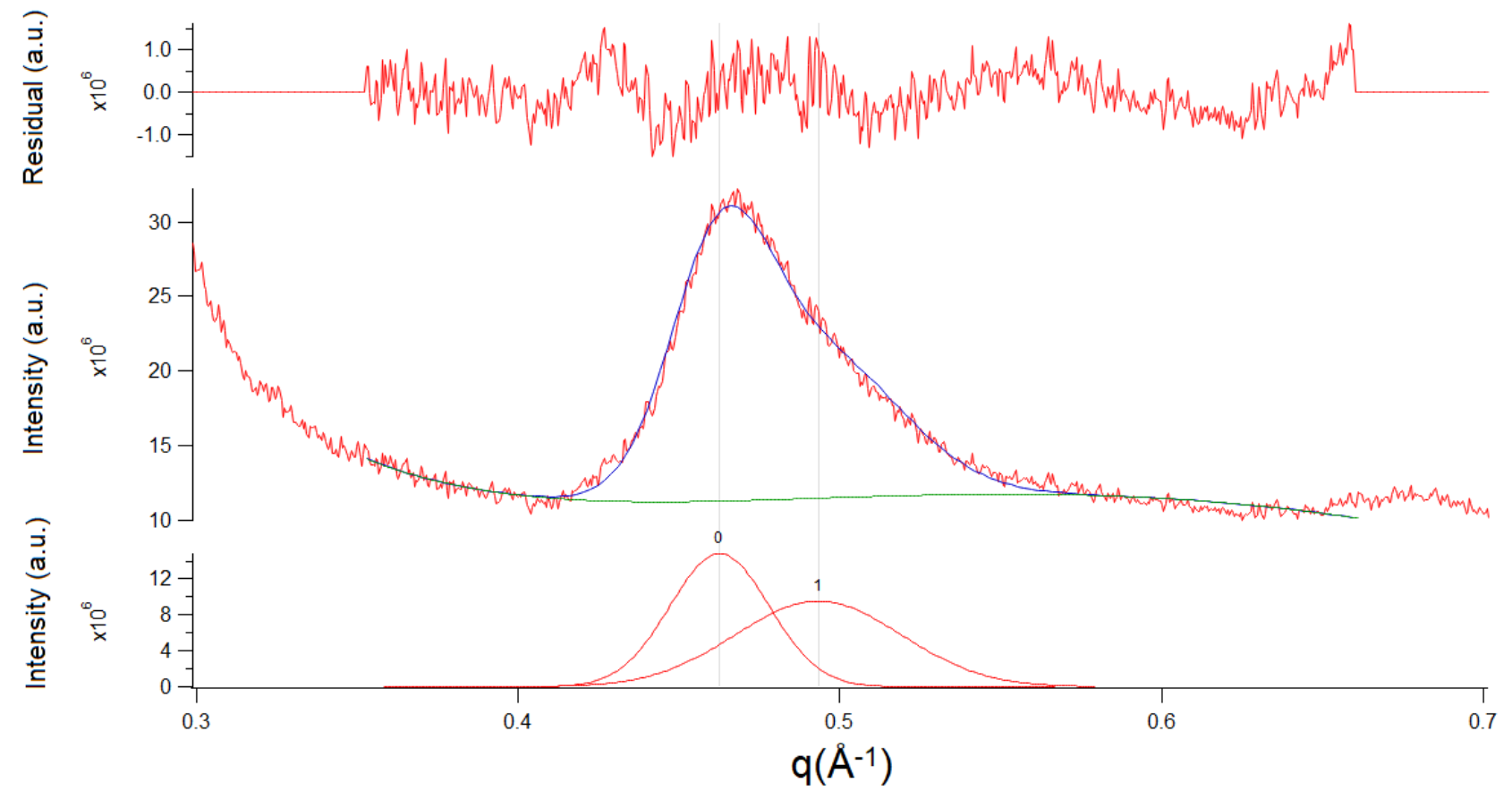

Figure $\mathbf{S}_{4}$. Example peak fit used separate the (oo1) peak and (200) peak. The same peak positions and peak widths were used for all energies with only the peak intensity allowed to vary. 


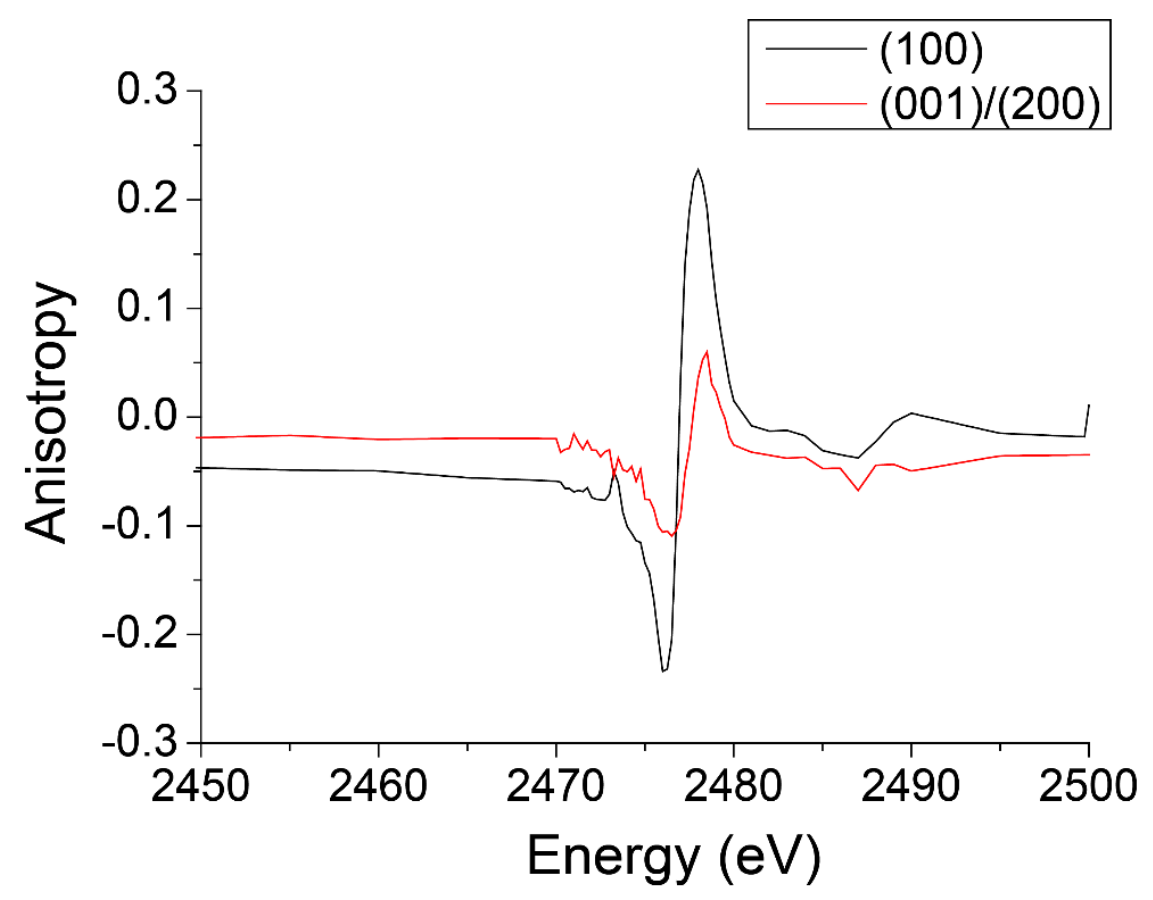

Figure $\mathbf{S}_{5}$. Line profiles of the energy dependence of anisotropy for the (100) and composite (oo1)/(200) peaks. 

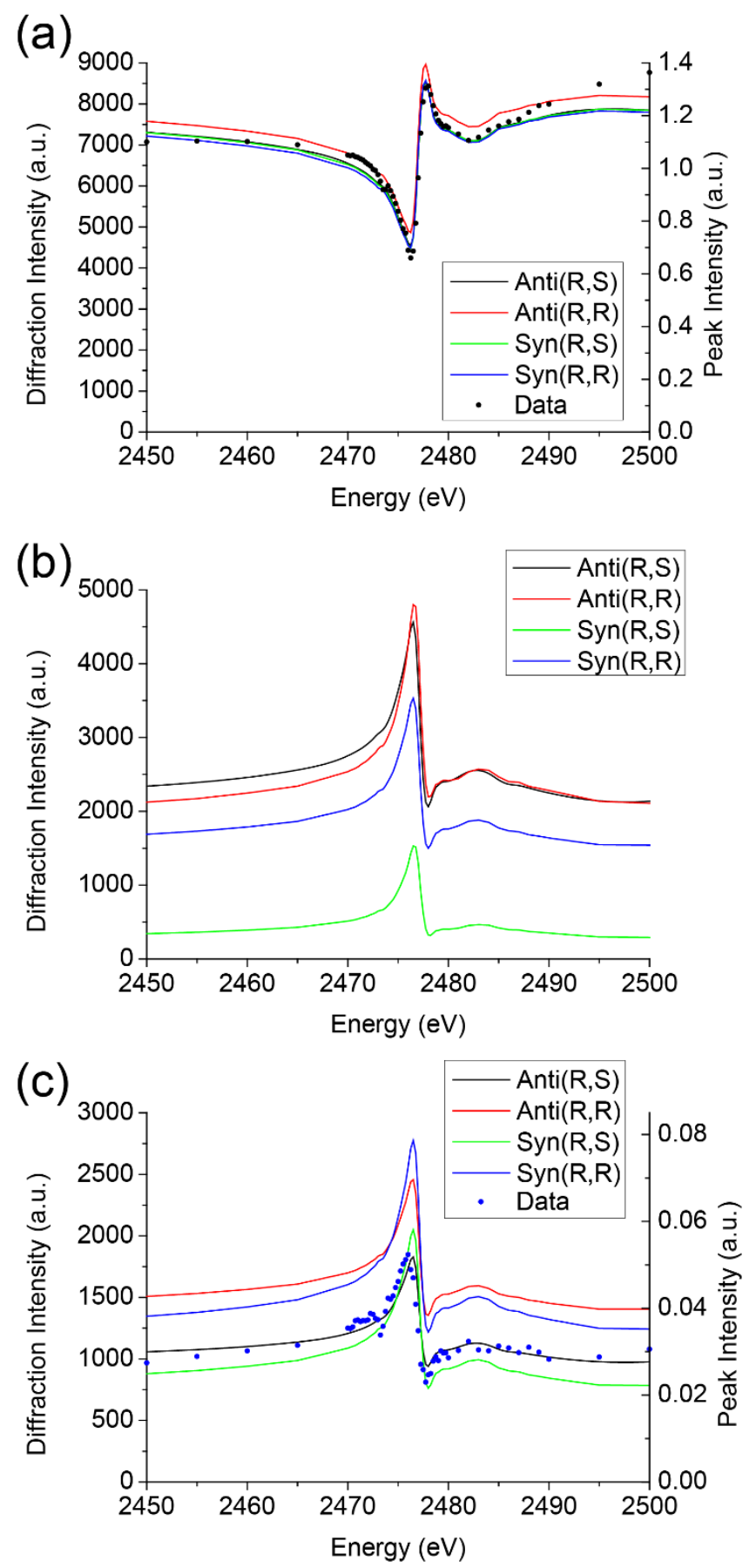

Figure S6. Simulated resonant diffraction profiles of the (a) (100) lamellar stacking peak, (b) (o10) $\pi$ stacking peak, (c) (oo1) backbone stacking peak, for the four different crystal structures of P(NDI2ODT2). Experimental data is not shown for the case of the (o10) peak as this peak is not experimentally accessible at $\sim 2.5 \mathrm{keV}$ (the scattering angle is too high). 

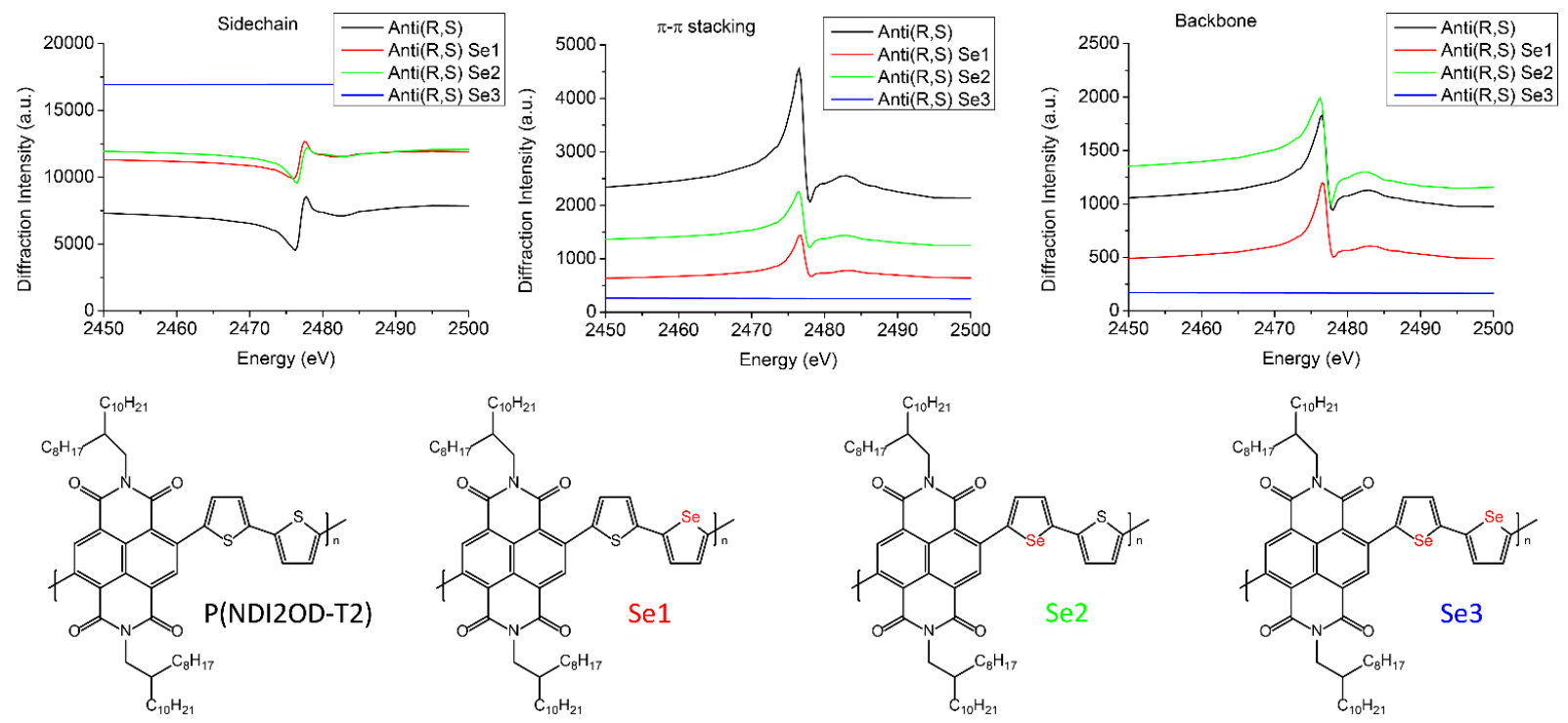

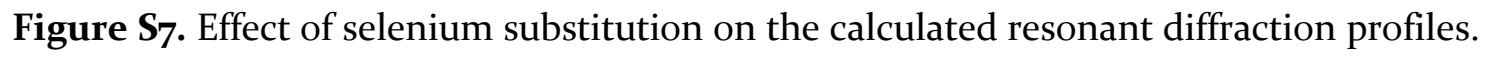



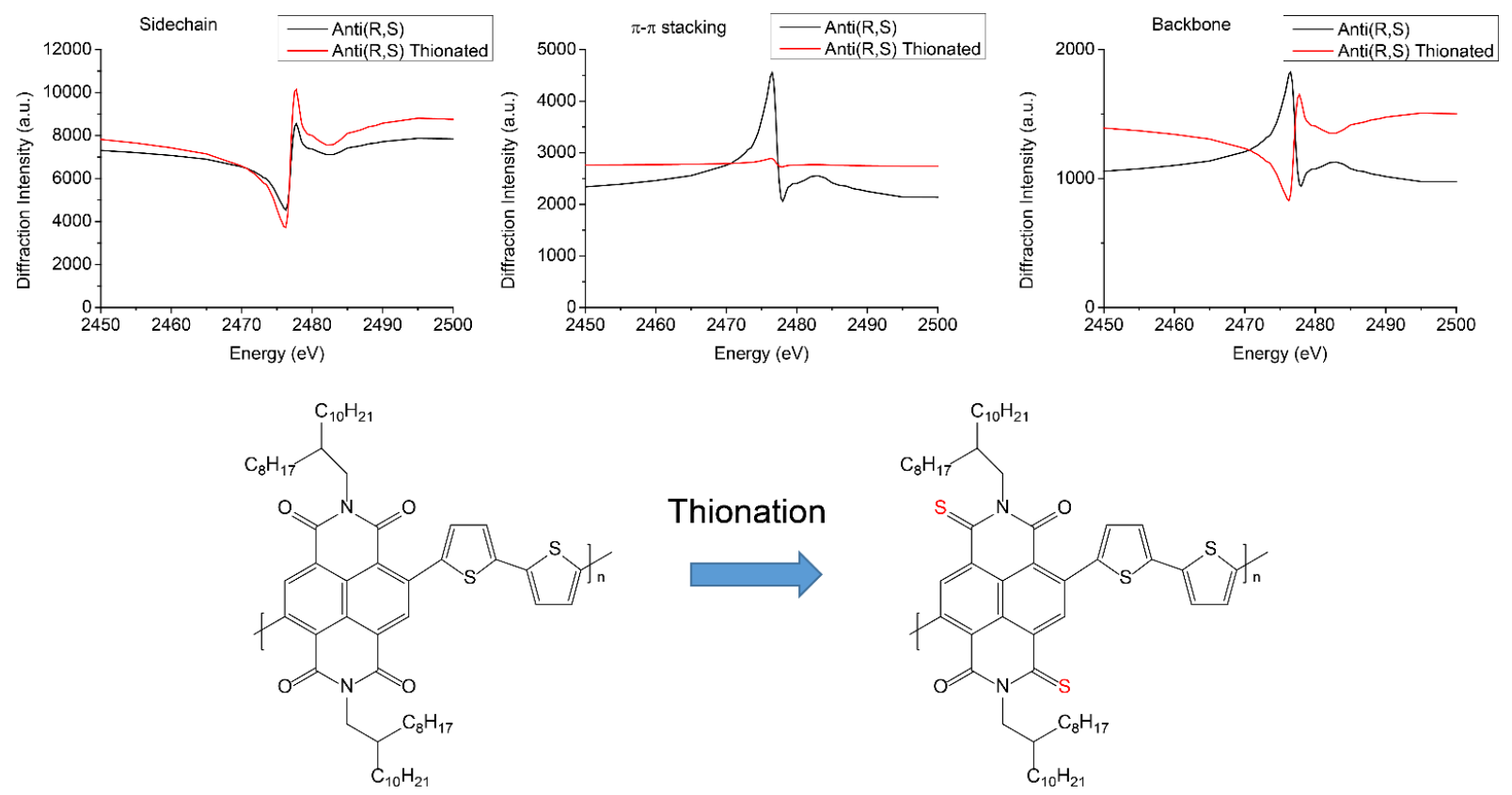

Figure S8. Effect of thionation on the calculated resonant diffraction profiles. 


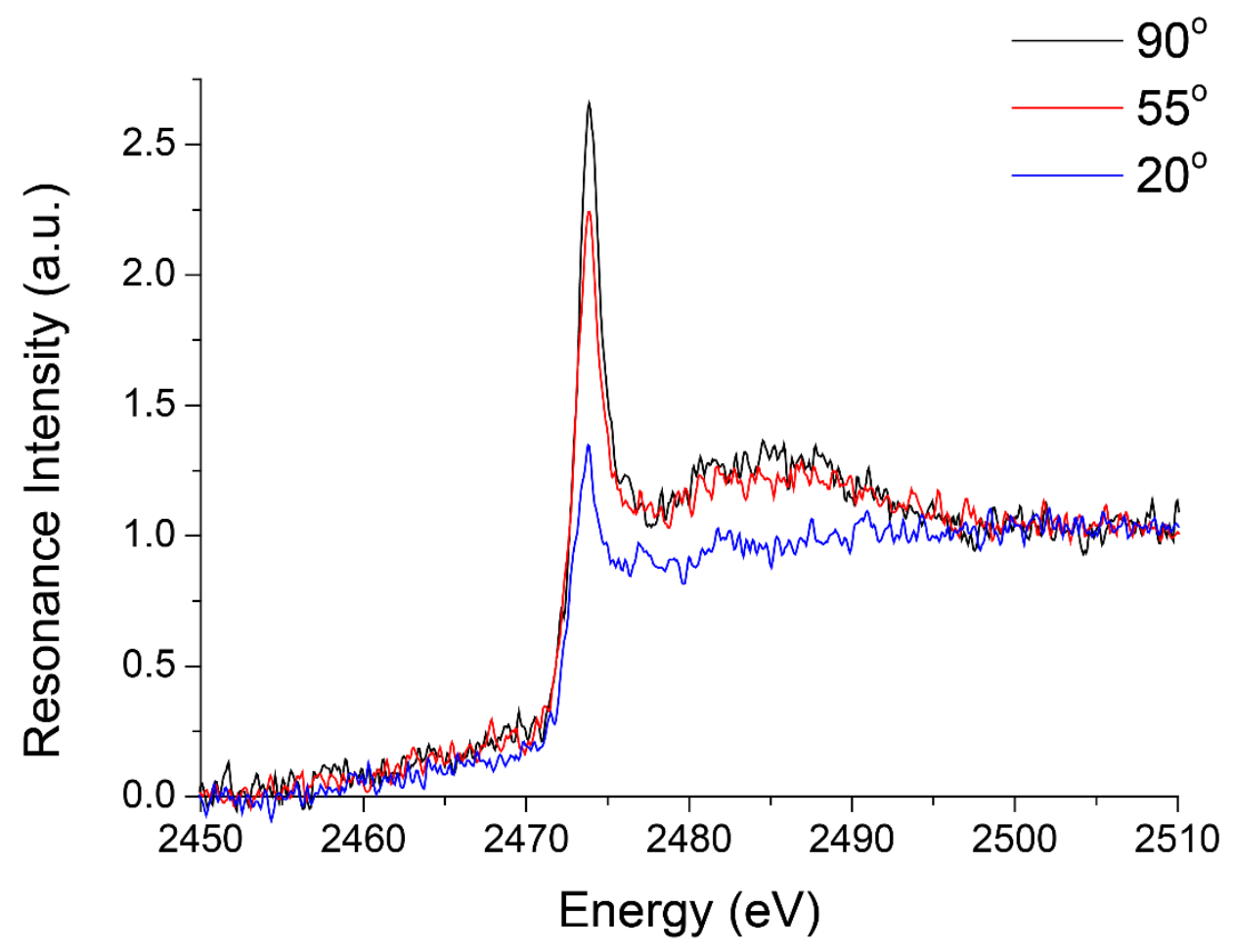

Figure S9. Angle-resolved NEXAFS spectra of P(NDI2OD-T2). 
Table S1. Summary of parameters used to fit the energy dependencies of the (100), (010), and (001) peaks calculated from the various models to Equation 4.

\begin{tabular}{|l|c|c|c|c|c|c|}
\hline & \multicolumn{2}{|c|}{$(100)$} & \multicolumn{2}{c|}{$(010)$} & \multicolumn{2}{c|}{$(001)$} \\
\hline & $\left|F_{0}\right| /\left|F_{\text {res }}\right|$ & $\Delta \varphi$ & $\left|F_{0}\right| /\left|F_{\text {res }}\right|$ & $\Delta \varphi$ & $\left|F_{0}\right| /\left|F_{\text {res }}\right|$ & $\Delta \varphi$ \\
\hline P(NDIOD-T2) & 2.11 & $0.0^{\circ}$ & - & - & 1.70 & $206.5^{\circ}$ \\
\hline Anti $(R, S)$ & 3.08 & $1.1^{\circ}$ & 1.40 & $183.0^{\circ}$ & 1.80 & $183.5^{\circ}$ \\
\hline Anti $(R, R)$ & 3.14 & $3.8^{\circ}$ & 1.23 & $162.0^{\circ}$ & 2.07 & $183.3^{\circ}$ \\
\hline Syn $(R, S)$ & 3.03 & $1.3^{\circ}$ & 0.34 & $189^{\circ}$ & 0.99 & $184.4^{\circ}$ \\
\hline Syn $(R, R)$ & 3.01 & $2.9^{\circ}$ & 1.24 & $181.7^{\circ}$ & 1.29 & $179.5^{\circ}$ \\
\hline Anti $(R, S)$ Se1 & 6.90 & $22.6^{\circ}$ & 1.26 & $158.8^{\circ}$ & 1.07 & $164.3^{\circ}$ \\
\hline Anti $(R, S)$ Se2 & 7.16 & $-11.5^{\circ}$ & 1.97 & $184.3^{\circ}$ & 2.00 & $209.2^{\circ}$ \\
\hline Anti $(R, S)$ Se3 & $\infty$ & - & $\infty$ & - & $\infty$ & - \\
\hline Anti $(R, S)$ Thionated & 2.11 & $1.0^{\circ}$ & 26.6 & $182.9^{\circ}$ & 2.87 & $1.2^{\circ}$ \\
\hline
\end{tabular}

